Laura Bogaert, Stefanie Van den Bremt, Sofie Schouwers, Xavier Bossuyt and Lieve Van Hoovels*

\title{
Harmonizing by reducing inter-run variability: performance evaluation of a quality assurance program for antinuclear antibody detection by indirect immunofluorescence
}

https://doi.org/10.1515/cclm-2018-0933

Received August 27, 2018; accepted December 5, 2018

\begin{abstract}
Background: The introduction of automated anti-nuclear antibody (ANA) indirect immunofluorescence (IIF) analysis may allow for more harmonized ANA IIF reporting, provided that a thorough quality assurance program controls this process. The aim of this study was to evaluate various quality indicators used for ANA IIF analysis with the final goal of optimizing the iQC program.
\end{abstract}

Methods: In an experimental setup, we introduced artificial errors, mimicking plausible problems during routine practice on a QUANTA-Lyser-NOVA View ${ }^{\circledR}$ system (Inova Diagnostics, San Diego, CA, USA). Predetermined quality indicators were evaluated against predefined acceptance criteria. In addition, we retrospectively investigated the applicability of the selected quality indicators in the daily routine practice during three pre-defined periods.

Results: Both the experimental as the retrospective study revealed that pre-analytical, analytical and post-analytical errors were not highlighted by company internal quality control (iQC) materials. The use of patient derived iQC samples, median fluorescence intensity results per run and the percentage of positive ANA IIF results as additional quality indicators ensured a more adequate ANA IIF quality assurance. Furthermore, negative and moderate positive sample IQC materials merit clinical validation, as titer changes of $>1$ correspond to clinically important

\footnotetext{
*Corresponding author: Lieve Van Hoovels, Department of Laboratory Medicine, OLV Hospital Aalst, Moorselbaan 164, 9300 Aalst, Belgium, Phone: +32 (0)53/72 42 91, Fax: +32 (0)53/72 45 88, E-mail: lieve.van.hoovels@olvz-aalst.be

Laura Bogaert: Department of Laboratory Medicine, OLV Hospital Aalst, Aalst, Belgium; and Department of Laboratory Medicine, GZA Hospitals, Antwerp, Belgium

Stefanie Van den Bremt: Department of Laboratory Medicine, OLV Hospital Aalst, Aalst, Belgium

Sofie Schouwers: Department of Laboratory Medicine, GZA Hospitals, Antwerp, Belgium

Xavier Bossuyt: Department of Laboratory Medicine, University Hospital Leuven, Leuven, Belgium; and Department of Microbiology and Immunology, KU Leuven, Leuven, Belgium
}

shifts. Traditional Westgard rules, including a clinically defined stop limit, revealed to be useful in monitoring of the supplemental quality indicators.

Conclusions: A thorough ANA IIF quality assurance for daily routine practice necessitates the addition of supplemental quality indicators in combination with welldefined acceptance criteria.

Keywords: antinuclear antibodies; automation; indirect immunofluorescence; quality control.

\section{Introduction}

The detection of anti-nuclear antibodies (ANA) is important in the diagnosis of antibody-associated rheumatic diseases (AARDs) [1, 2]. The gold standard screening method for ANA, indirect immunofluorescence assay (IIF) on human epithelial (HEp-2) cells [3], is hampered by a number of disadvantages like a high workload, subjective visual reading and a high intra- and inter-laboratory variance [4-6]. Most of the recent ANA IIF guidelines recommend not only to report the presence of ANA as positive or negative, but also to give an ANA IIF pattern together with a semi-quantitative result $[7,8]$. Through the introduction of automated microscopic analysis more harmonized ANA IIF reporting becomes feasible [9]. The latter was confirmed by a Belgian multicenter study among laboratories performing ANA detection by NOVA View ${ }^{\circledR}$ (Inova Diagnostics, San Diego, CA, USA), demonstrating a lower ANA IIF titer variability in comparison to manual ANA IIF [10]. However, for two laboratories that participated in the multicenter study clinically important deviations were found due to pre-analytical (malfunction of the slide processor) and analytical problems (a shift caused by instrument calibration and conjugate lot switch). The local quality assurance program did not detect these issues. In order to realize harmonization in ANA testing a quality assurance program monitoring the total testing process and detecting clinically significant shifts of ANA IIF measurements $[11,12]$ is a conditio sine qua non. The latter should cover all steps of the process (from the pre- to the post-analytical phase [13]). Appropriate iQC materials should be selected 
with relevant target values and be supported by welldefined quality indicators and $\mathrm{QQC}$ acceptance criteria. Adequate procedures for validation of instrument/reagent calibration and lot switches should be in place. With their ability to report fluorescence intensity as (semi-) quantitative results, automated ANA IIF systems have an excellent tool to define objective acceptance criteria supporting a quality assurance program.

In addition, further efforts should be undertaken to harmonize automated IIF analysis [9, 10]. This could include the use of universal standards for the calibration of the instruments and monitoring of the quality of the slides and reagents.

In this study we assess the performance of an iQC program designed for the QUANTA-Lyser-NOVA View ${ }^{\circledR}$ system (Inova Diagnostics, San Diego, CA, USA). Although the use of nominal light intensity units (LIU) values is not intended for IQC purposes as defined by the company, we investigate whether LIU could be integrated as a tool for analytical process validation. Quality indicators and iQC acceptance criteria were defined and evaluated in two phases: first in an experimental setup, mimicking plausible errors in routine ANA IIF practice, and secondly, in daily routine laboratory practice.

\section{Materials and methods}

\section{QUANTA-Lyser-NOVA View ${ }^{\circledR}$ system}

The QUANTA-Lyser 2 instrument (Inova Diagnostics, San Diego, CA, USA) is a pre-analytical platform for ANA IIF which automatically performs sample dilution and HEp-2 slide (NOVA Lite HEp-2 ANA kit, Inova Diagnostics, San Diego, CA, USA) processing according to the instructions of the manufacturer. NOVA View ${ }^{\circledR}$ is an automated fluorescent microscope programmed to acquire, archive and manage digital images of fluorescent stained slides. The system encloses an Olympus $1 \times 81$ inverted IIF microscope with $4 \times, 10 \times$ and $40 \times$ objectives and dual band DAPI/FITC/HC filters, computer, LED light source and a Kappa DX4 digital camera. The LED UV light source is a CoolLed PreciseExcite with excitation wavelengths of $400 \mathrm{~nm}$ (DAPI) and $490 \mathrm{~nm}$ (FITC). DAPI fluorescence is used by the NOVA View ${ }^{\circledR}$ software for localizing the HEp-2 cells and focusing. Thereafter, the image analysis is performed based on the FITC signal. For each well on a slide, three to eight images (depending on the laboratory specific settings) are acquired with both the DAPI and the FITC filter. Using FITC images, the system measures the average intensity in units, named as LIU, enabling the discrimination between positive and negative samples. The cut-off set by Inova for ANA IIF positivity is 48 LIU. The NOVA View ${ }^{\circledR}$ is able to identify and propose five basic fluorescent ANA patterns (homogeneous, speckled, centromere, nucleolar and nuclear dots) based on software algorithms. Using pattern-specific dilution curves, the measured LIU can be converted in an estimated endpoint titer (single well titer [SWT]) [13].

\section{IQC materials and quality indicators}

In addition to the company iQC materials of the NOVA Lite HEp-2 ANA kit, routine patient samples were selected as supplemental iQC material (further referred to as 'sample iQC'). For the negative sample iQC, anonymized negative ANA IIF (AC-0) [14] rest samples were pooled. For the positive sample iQC, two different pools were made, one with a speckled ANA IIF pattern (AC-4) and one with a homogeneous pattern (AC-1), both targeting a titer of 200 LIU, resulting in a corresponding SWT of 1:160.

Next to the IQC materials, 16 routine ANA IIF left-over samples were included. Those samples were retrospectively selected, to simulate a real-life routine ANA IIF run in terms of positive/negative ratio and distribution of the different LIU-values and corresponding SWT (Supplementary Data Table 1). For each of these samples a reference LIU was obtained during an ANA IIF run under perfectly controlled circumstances.

Median patient sample LIU per run and percentage ANA IIF positive patient samples per run were defined as quality indicators.

All serum samples were obtained as part of routine screening for ANA and anonymized after selection. No informed consent was needed for this retrospective study, but the study was performed according to the Declaration of Helsinki.

\section{Acceptance criteria}

Based on the total imprecision of the sample iQC materials, analyzed on the QUANTA-Lyser - NOVA View ${ }^{\circledR}$ system in accordance to the CLSI EP5-A2 protocol (for the negative (mean LIU < 49), positive speckled (mean LIU $=189.4$ ) and positive homogeneous sample iQC (mean LIU $=264.8$, CV\% of, respectively $37 \%, 27 \%$ and 26\%; cfr. Supplementary data Table 2 and Supplementary data Figure 1) [15], we defined a target CV for the iQC of $25 \%$. Westgard rules $1_{2 \mathrm{CV}}$ (a single control measurement exceeds the mean $+/-2^{\star} \mathrm{CV} \%$ target) and $1_{3 \mathrm{CV}}$ (a single control measurement exceeds the mean $+/-3{ }^{\star} \mathrm{CV} \%$ target) were applied as, respectively, warning and stop limit. The lower limit of the stop range for the positive speckled sample iQC, corresponded to a clinically relevant change of $>1$ ANA IIF titer (target: 1:160; $>3 \mathrm{CV} \%$ : $<1: 80$ ). For a positive sample with LIU around 200 and corresponding SWT of 1:160, a decrease of $>3^{\star} \mathrm{CV} \%$, can result in a LIU below the diagnostics cut-off (LIU 48) and a change in titer to $<1: 80$. An overview of the target values and acceptance criteria for the positive and negative $\mathrm{QQC}$ is given in Table 1.

These acceptance criteria were also adopted for the quality indicators.

\section{Artificial induced errors}

Artificial errors, mimicking plausible errors in routine ANA IIF practice, were introduced during the ANA IIF analytical process (one run per error). An overview of these artificial errors is listed in Table 2. For each of these runs the pattern, LIU and SWT results of the iQC materials and patient samples were recorded. $\mathrm{QQC}$ results were assessed according to the Westgard rules and patient results were compared to the pre-set target values and used to calculate the quality indicator (median LIU and \% positive). 
Table 1: Acceptance criteria for all quality indicators.

\begin{tabular}{|c|c|c|}
\hline & Target value and coefficient of variation (CV) & Acceptance criteria \\
\hline \multicolumn{3}{|l|}{ Process control } \\
\hline LIU positive kit iQC & $\begin{array}{l}\text { - Pattern of initial ANA IIF' analysis } \\
\text { - Target CV: } 25 \%\end{array}$ & $\begin{array}{l}\text { - Exact match of target pattern } \\
\text { - Westgard rules }\end{array}$ \\
\hline LIU $^{\mathrm{a}}$ negative kit iQC ${ }^{\mathrm{b}}$ & $\begin{array}{l}\text { - Negative on } 1 / 80 \text { dilution } \\
\text { - Target CV: } 25 \%\end{array}$ & $\begin{array}{l}\text { - Negative } \\
\text { - Westgard rules }\end{array}$ \\
\hline $\begin{array}{l}\text { LIU positive sample } \mathrm{IQC}^{\mathrm{b}} \text { (speckled/ } \\
\text { homogeneous) }\end{array}$ & $\begin{array}{l}\text { - Pattern of initial ANA IIFc analysis } \\
\text { - Target LIU } \text {-value: }+ \text { / - } 200 \\
\text { - Target CV: } 25 \%\end{array}$ & $\begin{array}{l}\text { - Exact match of target pattern } \\
\text { - Westgard rules }\end{array}$ \\
\hline LIUa negative sample iQC ${ }^{b}$ (patient pool) & $\begin{array}{l}\text { - Negative on } 1 / 80 \text { dilution } \\
\text { - Target LIU } \text {-value: }<48 \\
\text { - Target CV: } 25 \%\end{array}$ & $\begin{array}{l}\text { - Negative } \\
\text { - Westgard rules }\end{array}$ \\
\hline \multicolumn{3}{|l|}{ Monitoring of patient results } \\
\hline$\%$ Positive ANA IIF' patient samples/run ${ }^{c}$ & $\begin{array}{l}\text { - Target value: positive/negative ratio of a real-life routine run } \\
\text { - Target CV: } 25 \%\end{array}$ & - Westgard rules ${ }^{d}$ \\
\hline Median patient sample LIU $^{\mathrm{a}} /$ run $^{c}$ & $\begin{array}{l}\text { - Target value: overall median of the } 16 \text { study patient samples } \\
\text { (distribution of the LIU }{ }^{\mathrm{a}} \text {-values of a real-life routine run) } \\
\text { - Target CV: } 25 \%\end{array}$ & - Westgard rules ${ }^{d}$ \\
\hline
\end{tabular}

aLIU, light intensity units; ${ }^{\mathrm{b}} \mathrm{QQC}$, internal quality control; 'ANA IIF, anti-nuclear antibodies indirect immunofluorescence test; 'only for screening dilution (1/80); ${ }^{\mathrm{d}}{ }_{2 \mathrm{CV}}$ (a single control measurement exceeds the mean $+/-2 \mathrm{CV} \%$ target) as a warning limit and $1_{3 \mathrm{CV}}$ (a single control measurement exceeds the mean $+/-3 \mathrm{CV} \%$ target) as stop limit.

Table 2: Overview of all errors introduced in the ANA IIF analytical process.

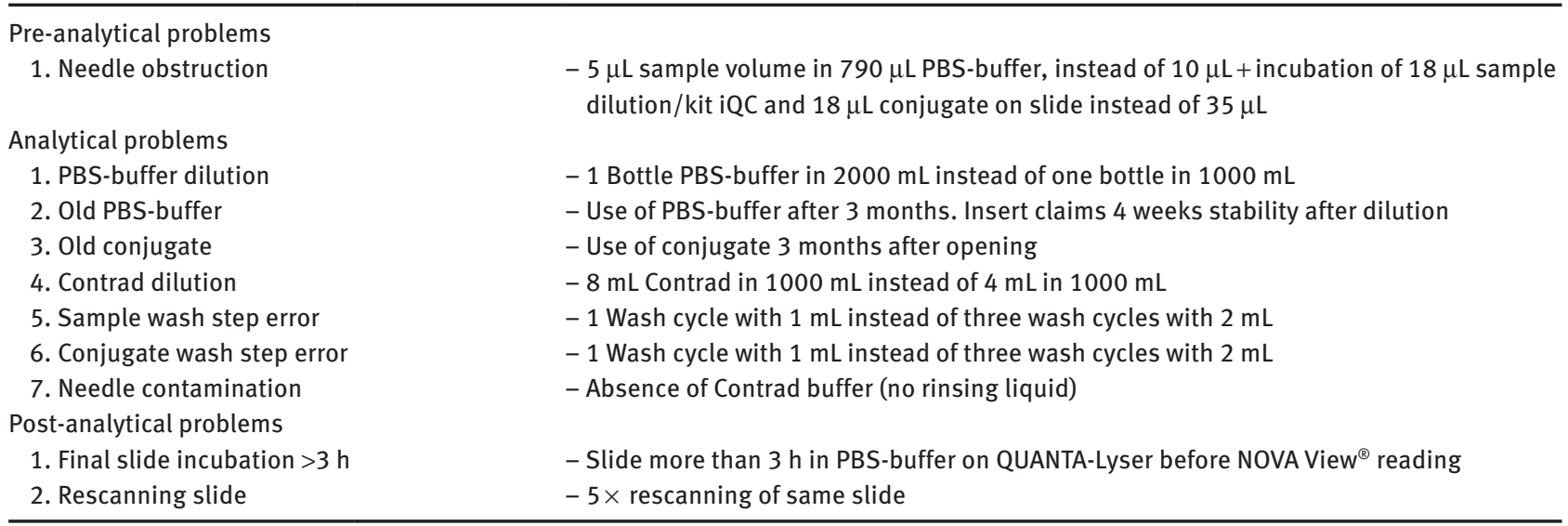

\section{Daily routine practice}

In order to evaluate the relevance and performance of the designed iQC program, the calculation of the quality indicators and the assessment of IQC results according to Westgard rules was retrospectively performed for three periods.

Period 1 was a stable period of 10 consecutive runs without any technical instrument intervention. Period 2 contained a technical intervention for the QUANTA-Lyser - NOVA View ${ }^{\circledR}$ system. Period 3 was derived from laboratory L6 of the Belgian multicenter study in 2016. Period 1 and period 2 included runs with a minimum of 20 patient samples per run and using the same lot number of NOVA Lite HEp-2 ANA kit and sample iQC materials.

\section{Results}

\section{Evaluation of ANA IIF quality indicators in a prospective experimental setup}

An overview of the results obtained from the different experiments is given in Table 3.

Three artificial errors had a manifest influence on the ANA IIF LIU results: (1) needle contamination; (2) needle obstruction; (3) incorrect dilution of the PBS buffer. In the case of needle obstruction, $88 \%(n=21 / 24)$ of the samples 
Table 3: Impact of each error on the different quality indicators.

\begin{tabular}{|c|c|c|c|c|c|c|c|}
\hline & \multicolumn{4}{|c|}{ Rescanning $5 x$} & \multirow{2}{*}{$\begin{array}{r}\text { Slide } \\
\text { incubation }>3 \mathrm{~h}\end{array}$} & \multirow{2}{*}{$\begin{array}{r}\text { Old conjugate } \\
3 \text { months }\end{array}$} & \multirow{2}{*}{$\begin{array}{r}\text { Needle } \\
\text { contamination }\end{array}$} \\
\hline & Scan 2 & Scan 3 & Scan 4 & Scan 5 & & & \\
\hline LIUa positive kit iQC $^{b}$ & $-11.1 \%$ & $-13.3 \%$ & $-19.7 \%$ & $-26.2 \%$ & $-9.4 \%$ & $-1.8 \%$ & $-0.8 \%$ \\
\hline LIU $^{\mathrm{a}}$ negative kit iQC & $0.0 \%$ & $0.0 \%$ & $0.0 \%$ & $0.0 \%$ & $0.0 \%$ & $0.0 \%$ & $0.0 \%$ \\
\hline LIUa positive sample iQC ${ }^{\mathrm{b}}$ speckled & $-35.1 \%$ & $-45.4 \%$ & $-50.2 \%$ & $-69.4 \%$ & $-40.5 \%$ & $-49.2 \%$ & $194.7 \%$ \\
\hline LIU ${ }^{\mathrm{a}}$ positive sample iQC ${ }^{\mathrm{b}}$ homogeneous & $-17.7 \%$ & $-49.6 \%$ & $-39.5 \%$ & $-63.2 \%$ & $-25.6 \%$ & $-56.6 \%$ & $134.9 \%$ \\
\hline LIU $^{\mathrm{a}}$ negative sample $\mathrm{iQC}^{\mathrm{b}}$ & $-16.7 \%$ & $-11.1 \%$ & $-33.3 \%$ & $-47.2 \%$ & $-11.1 \%$ & $-44.4 \%$ & $1276.0 \%$ \\
\hline$\%$ positive ANA IIFC Patient samples/run & $-8.6 \%$ & $-4.0 \%$ & $-10.0 \%$ & $-10.0 \%$ & $0.0 \%$ & $-10.0 \%$ & $50.0 \%$ \\
\hline \multirow[t]{2}{*}{ Median patient sample LIUa/run } & $-14.9 \%$ & $-41.5 \%$ & $-54.9 \%$ & $-65.9 \%$ & $-24.7 \%$ & $-60.1 \%$ & $166.6 \%$ \\
\hline & \multicolumn{2}{|c|}{$\begin{array}{r}\text { Needle } \\
\text { obstruction }\end{array}$} & $\begin{array}{l}\text { Contrad } \\
\text { dilution }\end{array}$ & $\begin{array}{r}\text { PBS buffer } \\
\text { dilution }\end{array}$ & $\begin{array}{r}\text { Sample } \\
\text { wash step }\end{array}$ & $\begin{array}{l}\text { Conjugate } \\
\text { wash step }\end{array}$ & Old buffer \\
\hline LIU $^{\mathrm{a}}$ positive kit iQC & \multicolumn{2}{|r|}{$1.4 \%$} & $6.2 \%$ & $-2.4 \%$ & $-10.5 \%$ & $-2.5 \%$ & $-4.2 \%$ \\
\hline LIU $^{\mathrm{a}}$ negative kit iQC ${ }^{\mathrm{b}}$ & \multicolumn{2}{|r|}{$0.0 \%$} & $0.0 \%$ & $0.0 \%$ & $0.0 \%$ & $0.0 \%$ & $0.0 \%$ \\
\hline LIUa positive sample iQC ${ }^{\mathrm{b}}$ speckled & \multicolumn{2}{|c|}{$-85.7 \%$} & $-30.4 \%$ & $105.9 \%$ & $-43.8 \%$ & $43.1 \%$ & $-8.1 \%$ \\
\hline LIU ${ }^{\mathrm{a}}$ positive sample iQC ${ }^{\mathrm{b}}$ homogeneous & \multicolumn{2}{|c|}{$-92.2 \%$} & $-33.1 \%$ & $123.6 \%$ & $-39.2 \%$ & $64.6 \%$ & $-12.8 \%$ \\
\hline LIU $^{\mathrm{a}}$ negative sample $\mathrm{IQC}^{\mathrm{b}}$ & \multicolumn{2}{|c|}{$-100.0 \%$} & $-25.0 \%$ & $1430.6 \%$ & $-22.2 \%$ & $1.9 \%$ & $1.9 \%$ \\
\hline$\%$ positive ANA IIFC Patient samples/run & \multicolumn{2}{|c|}{$-62.5 \%$} & $0.0 \%$ & $60.0 \%$ & $10.0 \%$ & $25.0 \%$ & $0.0 \%$ \\
\hline Median patient sample LIUa/run & \multicolumn{2}{|c|}{$-86.0 \%$} & $-33.2 \%$ & $303.4 \%$ & $-37.5 \%$ & $89.1 \%$ & $-9.1 \%$ \\
\hline
\end{tabular}

Results are expressed as relative deviation in LIU from the target values (iQC) or reference run (median LIU/run and \% positive/run), with LIU changes exceeding the warning limits highlighted in italic and changes exceeding the stop limits in italic and bold. aLIU, light intensity units; biQC, internal quality control; 'ANA IIF, anti-nuclear antibodies indirect immunofluorescence test.

revealed a relative change in LIU of more than $2^{\star} \mathrm{CV}(50 \%)$ and even $79 \%(n=19 / 24)$ of the samples had a relative difference of more than $3^{\star} \mathrm{CV}(75 \%)$. Using an extra diluted PBS buffer increased the LIU of $75 \%$ of the samples more than $3^{\star} \mathrm{CV}$ compared to the reference LIU. Simulating needle contamination resulted in a relative change in LIU of more than $75 \%$ compared to the reference LIU for $67 \%$ $(n=16 / 24)$ of the test samples. For every error described above the LIU of the positive iQC sample (both homogeneous and speckled) and of the negative iQC sample exceeded the stop limit. Also, the median LIU exceeded the stop limit; the percentage of ANA IIF positive patients per run exceeded the predefined warning limit. Of note, LIU values of neither the positive nor the negative kit iQC exceeded the warning limits.

Wash cycle reduction after conjugate incubation also had an impact on the ANA IIF LIU of the patient samples. The LIU of $58 \%(n=14 / 24)$ of the samples changed relatively more than $2^{\star} \mathrm{CV}(50 \%)$ compared to the reference LIU, with even $25 \%(n=6 / 24)$ of the samples changing more than $3^{\star} \mathrm{CV}(75 \%)$. The evaluation of the positive and negative kit iQC revealed no problem, in contrast to the median LIU that exceeded the predefined stop limit $\left(>3^{\star} \mathrm{CV}\right)$. All other quality indicators remained within warning limits $\left(<2^{\star} \mathrm{CV}\right)$.

The ANA IIF LIU of almost half of the patient samples was reduced by using an old conjugate and rescanning the slide 5 times, but no stop limit was exceeded. The same trend was observed for the positive sample iQC and the median LIU.

For all other artificially introduced errors (old PBS buffer, concentrated Contrad solution, wash cycle reduction after sample incubation and delayed IIF reading), no significant difference in ANA IIF LIU could be observed for the study samples and none of the evaluated quality indicators exceeded warning or stop limits.

\section{Retrospective evaluation of ANA IIF quality indicators in daily routine laboratory practice}

The imprecision data obtained by a retrospective analysis of the IQC data and quality indicators for 10 consecutive, stable routine ANA IIF runs are shown in Table 4 and Figure 1A. In this period, the positive and negative kit iQC revealed a low and a high imprecision, respectively. As the negative kit iQC only generates LIU results of ' 0 ' or ' 1 ', a high CV\% was to be expected. The variation of the positive speckled sample iQC (CV 34.7\%) exceeded the variation found in the experimental setup (CV 27.1\%) and was more in concordance with the $\mathrm{CV} \%$ of the negative sample iQC (CV 33.1\%). Our retrospective analysis revealed a relatively high variation (CV 47.1\%) for the median patient sample LIU per run. 
Figure $1 \mathrm{~B}$ and $\mathrm{C}$ show a graphical representation of the imprecision data obtained during a period with technical issues in two different laboratories.

Figure 1B describes a decreasing trend for all ANA IIF quality indicators except for the kit iQC during a 2-week period (period 2). A root cause analysis was initiated after the stop limit of $3^{\star} \mathrm{CV}(75 \%)$ was exceeded (May 23rd). Different corrective actions (needle replacement and extensive flushing) remained unsuccessfully.
Eventually, a technical intervention by the company revealed a reduction of the LED source intensity. After recalibration of the LED source, all quality indicators were restored to normal. During this whole period, the traditional positive and negative kit $\mathrm{iQC}$ remained normal.

Figure 1C shows the quality indicator data that could be retrieved from L6 during the Belgian multicenter study in 2016 [13]. The study revealed for L6 a remarkably larger

A

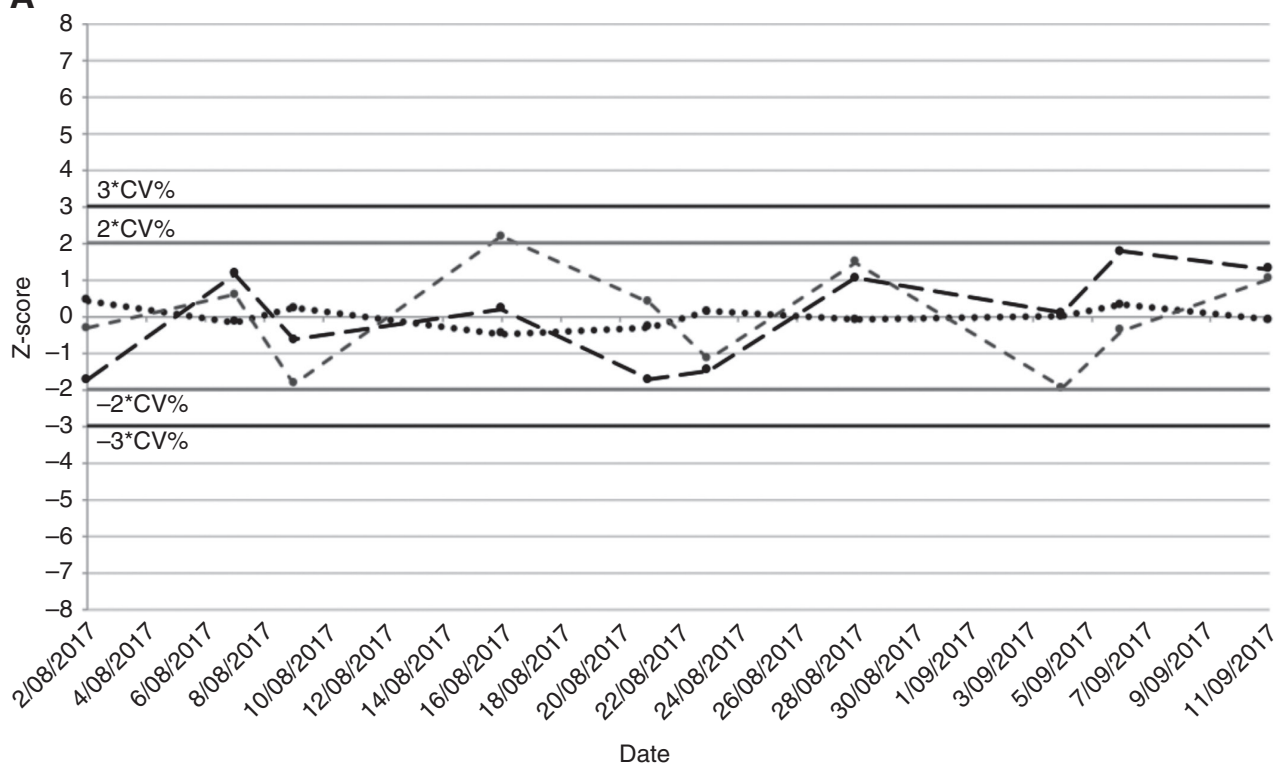

•.•-LIU pos kit iQC

(target LIU 2089)

- - LIU pos sample iQC SP (target LIU 270)

$\rightarrow$-LIU neg sample iQC (target LIU 33)

$1 \mathrm{CV}=25 \%$

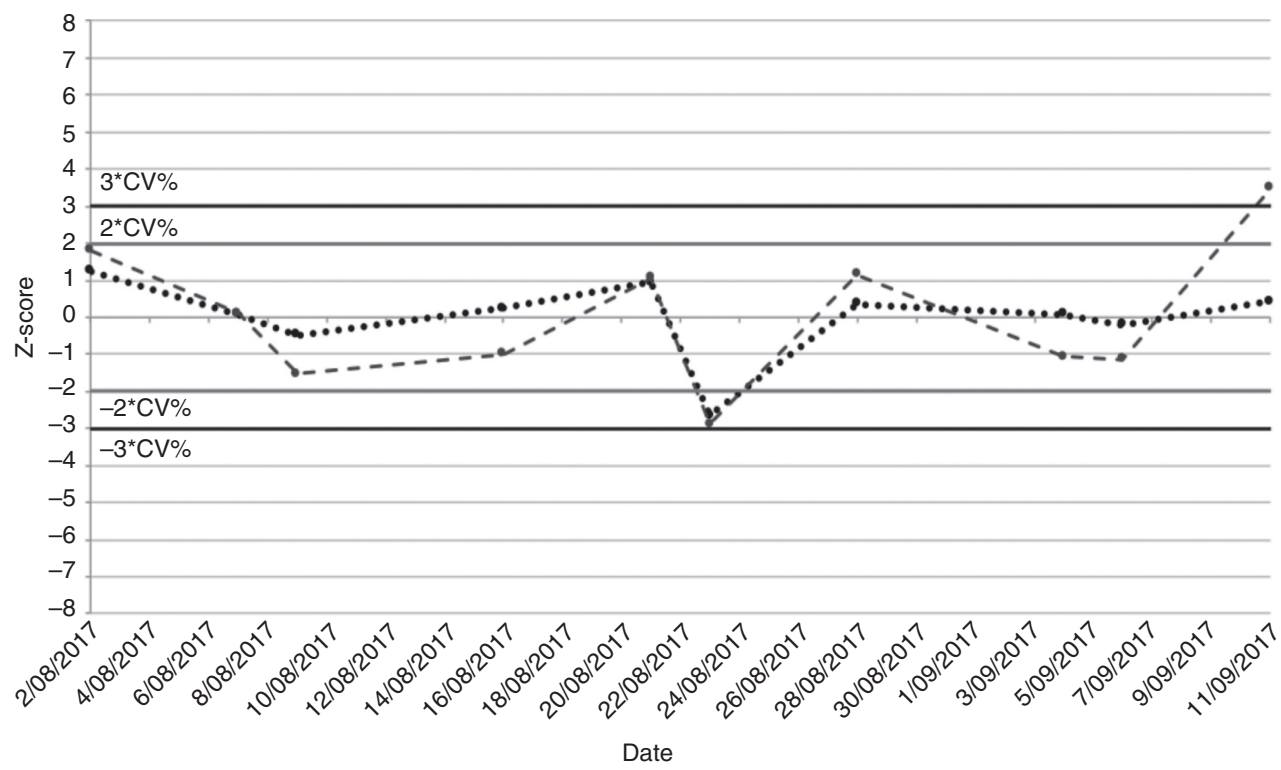

•..\% positive ANA IIF patient samples/run (target 59\%)

\section{- Median patient} sample LIU/run (target LIU 79)

$1 \mathrm{CV}=25 \%$

Figure 1: Levey-Jennings graph for the different quality indicators.

(A) Period 1: consecutive series $(n=10)$ of stable ANA IIF runs with no iQC violations (Lab 1). (B) Period 2: consecutive series ( $n=19)$ of ANA IIF runs during a 2-week period with a technical error concerning the LED light source. During root cause analysis different corrective actions were undertaken (marked by arrows) (Lab 1). (C) Period 3: data from 26 ANA IIF runs performed in L6 before, during and after the Belgian multicenter study in which a problem concerning the wash module of the slide processor was revealed [13]. 

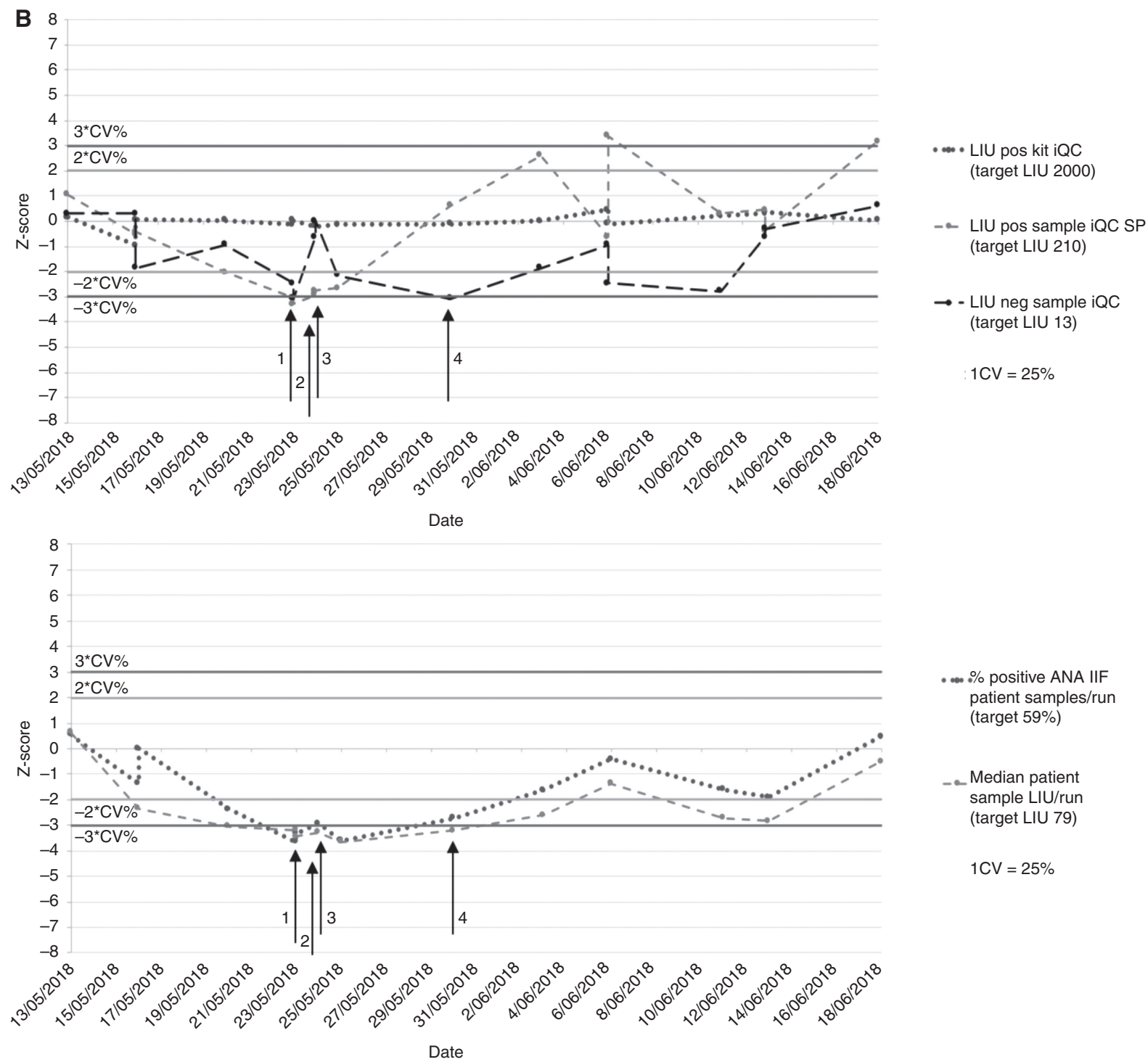

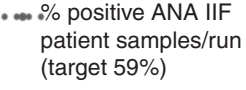

$1 \mathrm{CV}=25 \%$

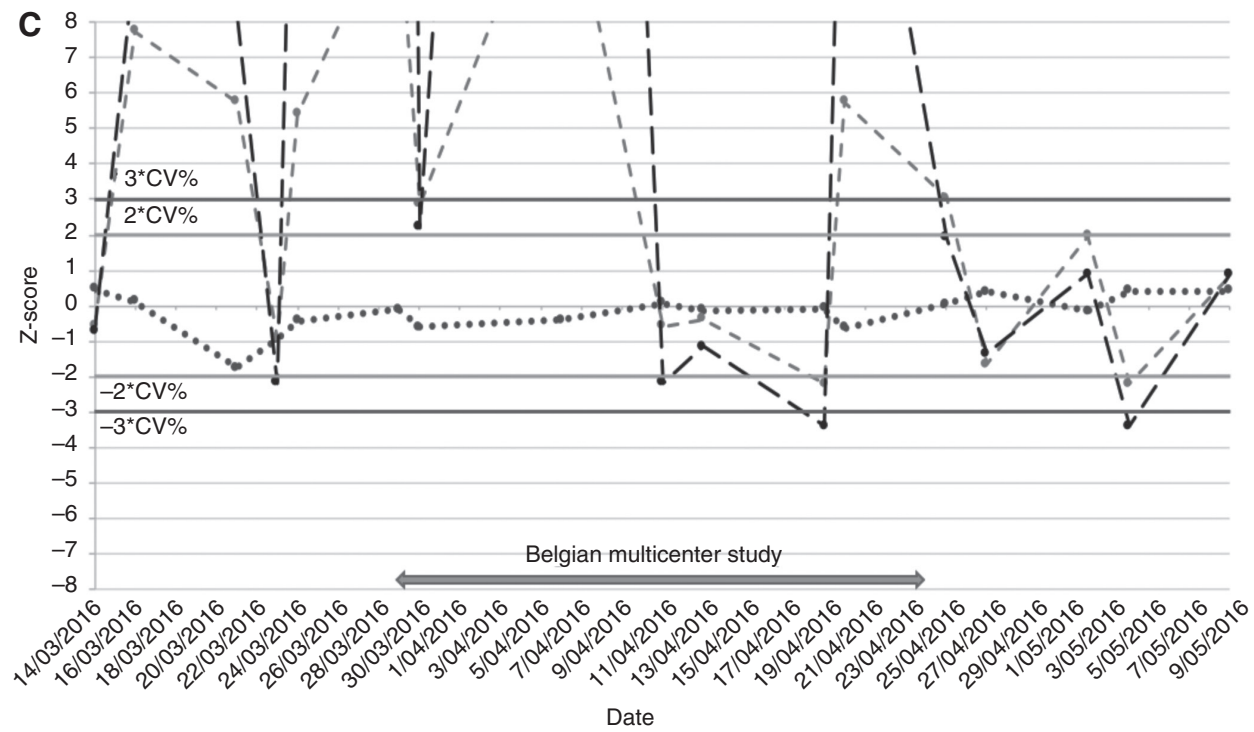

\footnotetext{
-.n. LIU pos kit iQC (target LIU 2245)

- $\%$ positive ANA IIF patient samples/run (target 22\%)

$\rightarrow$ - Median patient sample LIU/run (target LIU 20)

$1 \mathrm{CV}=25 \%$
}

Figure 1: (continued) 
ANA IIF LIU variation and higher median LIU values compared to the other laboratories. This was due to a problem of the washing module of the pre-analytical slide processor [13]. As shown in Figure 1C, these problems were not highlighted by the routine positive kit iQC. At that time the laboratory did not use sample iQCs. However, the $\%$ positive ANA IIF samples and the median LIU were markedly increased during the period of the multicenter study. After technical intervention of the slide processor of L6, new study samples were re-analyzed and showed a good concordance with the other laboratories and an improved inter-run CV [13].

\section{Discussion}

ANA IIF analysis intrinsically bears important analytical variables (e.g. lot number related differences in substrate and conjugate, the subjective result interpretation), which contribute to the large inter-assay variability $[5,9]$. The replacement of the IIF-based ANA screening test by solid phase assays (SPA) has been topic of debate since the initial recommendation by the American College Rheumatology (ACR) Task Force in 2010. However, advantages and disadvantages of the two methodological platforms have been reviewed and discussed, and none of the two immunoassays appears to completely satisfy the required demands [6]. Nowadays, the combination the ANA IIF as a screening assay and the use of new antigen-specific multiplex immunoassays in the context of a specific clinical setting, optimizes the diagnostic power [16-21]. Nevertheless, the shortcomings of the ANA IIF assay indisputably urge attention.

The aim of the implementation of automated ANA IIF microscopes, was to reduce the workload on the one hand, and to harmonize the ANA IIF analytical process on the other hand [5, 9, 22, 23]. Unfortunately, recent multicenter studies showed that even with the automated ANA IIF microscopes, ANA testing in clinical practice remains challenging [10-13]. Besides the persisting large inter-assay variability inherent to the analysis, the study of Van den Bremt et al. also revealed pre-analytical (problems with the washing unit of the pipetting system) and analytical (calibration) errors, which were not uncovered by the IQC program in place. IQC programs should never rely solely on the kit iQC materials. For a robust program internal quality material should be added [13]. The routinely obtained CV\% results of the kit iQC materials (Table 4) revealed that both the positive kit iQC (low CV\%) and negative kit iQC (high CV\%) are of little significance as a quality indicator. This was confirmed by the experimental set up as well as by the retrospective survey, in which none of the (artificially introduced) errors was highlighted by an IQC violation of the LIU of the kit iQC materials. To perform adequate quality assurance of the daily routine ANA IIF by automated instruments, additional quality indicators covering the entire ANA IIF process are necessary. Herein, the selection of adequate control materials is the most critical aspect in getting a thorough quality assurance program. First, it is important that the IQC material assures the whole ANA IIF process, from dilution up to result interpretation, which is not always the case for company iQC, which in many cases are 'ready for use' and do not require pre-dilution as routine patient samples do. Secondly, variations in LIU of patientderived $\mathrm{QQC}$ materials revealed an important contribution to the whole quality assurance process, as titer changes of $>1$ correspond to clinically important shifts [23]. From this perspective, negative and moderate positive sample iQC materials (LIU +/ - 200, for speckled and homogeneous IIF patterns corresponding to an ANA IIF titer of 1:160) revealed useful information. For positive sample iQC material, samples with an isolated homogeneous or speckled ANA IIF pattern are preferred, as they show the best inter-assay variability [24].

In addition, adequate procedures to ensure validation of calibration (reagent/instrument) and reagent lot switches should be in place.

To evaluate the predefined acceptance criteria and quality indicators of the experimental study with daily

Table 4: Retrospective imprecision analysis of quality indicators during routine ANA IIF analysis during a stable period.

Imprecision quality indicators (10 stable routine runs)

LIUa Positive kit LIUa Negative kit LIUa Positive sample LIUa Negative \% Positive ANA IIF ${ }^{\text {Median patient }}$ IQC $^{\mathrm{b}} \quad$ IQC $^{\mathrm{b}} \quad$ IQC $^{\mathrm{b}}$ speckled sample $\mathrm{iQC}^{\mathrm{b}}$ patient samples/run sample LIU $\mathrm{d} / \mathrm{run}$

\begin{tabular}{|c|c|c|c|c|c|c|}
\hline Mean & 2089.2 & 0.2 & 269.6 & 33.3 & 0.6 & 79.1 \\
\hline Standard deviation & 148.0 & 0.4 & 93.5 & 11.0 & 0.2 & 37.3 \\
\hline Coefficient of variation, $\%$ & $7.1 \%$ & $210.8 \%$ & $34.7 \%$ & $33.1 \%$ & $26.4 \%$ & $47.1 \%$ \\
\hline
\end{tabular}

aLIU, light intensity units; ${ }^{\mathrm{b}} \mathrm{QQC}$, internal quality control; 'ANA IIF, anti-nuclear antibodies indirect immunofluorescence test. 
practice, routinely-derived $\mathrm{iQC}$ data were retrospectively investigated. In general, the variation of most quality controls exceeded the variation found in the experimental setup. This finding confirms the persisting high inter-assay variability inherent to the ANA IIF analysis despite automation in the pre-analytical (QUANTA Lyser) and analytical (NOVA View ${ }^{\circledast}$ ) phase and underlines the need for more efforts in harmonizing automated ANA IIF analysis [9]. However, the evaluation of the different quality indicators in the experimental setup revealed that the follow-up of LIU values by applying traditional Westgard multi-rules $\left(1_{2 \mathrm{CV}}\right.$ as a warning limit and $1_{3 \mathrm{CV}}$ as stop limit) assisted in the analytical and clinical assurance of an ANA IIF run. Nevertheless, the high intrinsic CV\% of the ANA IIF analysis does not allow for the application of the $2^{\star} \mathrm{CV}(50 \%)$ for the FI of sample iQC materials as a quality control limit. At the most, this $2^{\star} \mathrm{CV}$ limit can be regarded as a warning signal and encourage iQC trend analysis. However, a clinically defined $75 \%$ stop limit should result in root cause analysis and review of acceptance of the whole ANA IIF analytical run, most definitely if different quality indicators are exceeding this limit at the same time. In the retrospective analysis of the two unstable periods, many of our predefined quality indicators exceeded the $1_{3 \mathrm{cv}}$-rule on the day or during the period the problem occurred, indicating that the accuracy of the ANA IIF results was no longer guaranteed.

As indicated by Maenhout and colleagues, it is worthwhile to include $\mathrm{IQC}$ monitoring based on whole run ANA IIF patient results in the daily routine iQC analysis [11]. The experimental study as well as the retrospective study revealed that the percentage ANA positive samples per run confirmed in most cases the findings of the other quality indicators. Both studies showed that the use of the median LIU of the patient samples per run yield relevant information. The introduction of an artificial error, which had an impact on patient results, was always accompanied by a violation of the median LIU of the patient samples per run. However, our retrospective analysis revealed a relatively high variability of the median patient sample LIU per run. Several $1_{3 \mathrm{CV}}$ stop limit violations of the median LIU per run were found over a period of time, without violation of any other quality indicator. Variations in demographic features of patients (e.g. age, gender, hospitalization status, clinical discipline of requester) involved in the ANA IIF analysis run, can be attributed to this large inter-run variability of the median LIU. A further refinement of median LIU calculations is warranted. Awareness is important so that in daily routine practice decisions are never taken by interpreting only one quality indicator. Furthermore, a thorough pre-analytical stability study should be performed to safeguard the stability of
iQC materials and a correct interpretation of the total iQC program. Finally, an extension of this study to other ANA IIF detection platforms, including automated systems but also manual procedures, is warranted.

In conclusion, our study revealed the necessity and feasibility of the implementation of extra quality indicators to ensure a thorough process control of automated ANA IIF analysis. Negative and moderate ANA IIF positive patient $\mathrm{iQC}$ samples evince the most useful information and make clinically quality acceptance of an ANA IIF run plausible.

Acknowledgments: We gratefully acknowledge Juul Boes (AZ Turnhout), Martijn van Zuijlen and Nathalie Vandeputte (Werfen N.V./S.A, Benelux) for their feedback and constructive collaboration. The results of this paper were presented as Critical Appraised Topic at the University Hospital Leuven as part of the training in Laboratory Medicine.

Author contributions: All the authors have accepted responsibility for the entire content of this submitted manuscript and approved submission.

Research funding: None declared.

Employment or leadership: XB has been consultant for Inova Diagnostics.

Honorarium: None declared.

Competing interests: The funding organization(s) played no role in the study design; in the collection, analysis, and interpretation of data; in the writing of the report; or in the decision to submit the report for publication.

\section{References}

1. Solomon DH, Kavanaugh AJ, Schur PH, American College of Rheumatology Ad Hoc Committee on Immunologic Testing Guidelines. Evidence-based guidelines for the use of immunologic tests: antinuclear antibody testing. Arthritis Rheum 2002;47:434-44.

2. Fritzler MJ. Choosing wisely: review and commentary on anti-nuclear antibody (ANA) testing. Autoimmun Rev 2016;15: 272-80.

3. Meroni PL, Schur PH. ANA screening: an old test with new recommendations. Ann Rheum Dis 2010;69:1420-2.

4. Rigon A, Infantino M, Merone M, Iannello G, Tincani A, Cavazzana I, et al. The inter-observer reading variability in anti-nuclear antibodies indirect (ANA) immunofluorescence test: a multicenter evaluation and a review of the literature. Autoimmun Rev 2017;16:1224-9.

5. Infantino M, Meacci F, Grossi V, Manfredi M, Benucci M, Merone $M$, et al. The burden of the variability introduced by the HEp-2 assay kit and the CAD system in ANA indirect immunofluorescence test. Immunol Res 2017;65:345-54. 
6. Meroni PL, Chan EK, Damoiseaux J, Andrade LE, Bossuyt $\mathrm{X}$, Conrad K, et al. Unending story of the indirect immunofluorescence assay on HEp-2 cells: old problems and new solutions? Ann Rheum Dis 2018 Apr 17; doi: 10.1136/annrheumdis-2018-213440.

7. Agmon-Levin N, Damoiseaux J, Kallenberg C, Sack U, Witte T, Herold $\mathrm{M}$, et al. International recommendations for the assessment of autoantibodies to cellular antigens referred to as antinuclear antibodies. Ann Rheum Dis 2014;73:17-23.

8. CLSI I/LA02-A2. Quality assurance of laboratory tests for autoantibodies to nuclear antigens: (1) Indirect Fluorescence Assay for Microscopy and (2) Microtiter Enzyme Immunoassay Methods; Approved guideline - Second edition. Wayne, PA: Clinical and Laboratory Standards Institute, 2006.

9. Mahler M. Lack of standardisation of ANA and implications for drug development and precision medicine. Ann Rheum Dis 2018 Mar 24; doi: 10.1136/annrheumdis-2018-213374.

10. Van Hoovels L, Schouwers S, Van den Bremt S, Bossuyt X. Variation in antinuclear antibody detection by automated indirect immunofluorescence analysis. Ann Rheum Dis 2018 Apr 20; doi: 10.1136/annrheumdis-2018-213543.

11. Maenhout TM, Bonroy C, Verfaillie C, Stove V, Devreese K. Automated indirect immunofluorescence microscopy enables the implementation of a quantitative internal quality control system for anti-nuclear antibody analysis. Clin Chem Lab Med 2014;52:989-98.

12. Mulliez SM, Maenhout TM, Bonroy C. Impact of the routine implementation of automated indirect immunofluorescence antinuclear antibody analysis: 1 year of experience. Clin Chem Lab Med 2016;54:e183-6.

13. Van den Bremt S, Schouwers S, Van Blerk M, Van Hoovels L. ANA IIF Automation: moving towards harmonization? Results of a multicenter study. J Immunol Res 2017;2017:6038137.

14. Andrade LE, Klotz W, Herold M, Conrad K, Rönnelid J, Fritzler MJ, et al. International consensus on antinuclear antibody patterns: definition of the AC-29 pattern associated with antibodies to DNA topoisomerase I. Clin Chem Lab Med 2018;56:1783-88.

15. CLSI EP5-A2. Evaluation of precision performance of quantitative measurement methods; Approved guideline - Second edition. Wayne, PA: Clinical and Laboratory Standards Institute, 2004.
16. Willems P, De Langhe E, Claessens J, Westhovens R, Van Hoeyveld E, Poesen K, et al. Screening for connective tissue disease-associated antibodies by automated immunoassay. Clin Chem Lab Med 2018;56:909-18.

17. Bossuyt X, Fieuws S. Detection of antinuclear antibodies: added value of solid phase assay? [Letter] Ann Rheum Dis 2014;73:e10.

18. Pérez D, Gilburd B, Azoulay D, Shovman O, Bizzaro N, Shoenfeld $Y$. Antinuclear antibodies: is the indirect immunofluorescence still the gold standard or should be replaced by solid phase assays? Autoimmun Rev 2018;17:548-52.

19. Bizzaro N, Brusca I, Previtali G, Alessio MG, Daves M, Platzgummer $\mathrm{S}$, et al. The association of solid-phase assays to immunofluorescence increases the diagnostic accuracy for ANA screening in patients with autoimmune rheumatic diseases. Autoimmun Rev 2018;17:541-7.

20. Claessens J, Belmondo T, De Langhe E, Westhovens R, Poesen $\mathrm{K}$, Hüe $\mathrm{S}$, et al. Solid phase assays versus automated indirect immunofluorescence for detection of antinuclear antibodies. Autoimmun Rev 2018;17:533-40.

21. Willems P, De Langhe E, Westhovens R, Vanderschueren S, Blockmans D, Bossuyt X. Antinuclear antibody as entry criterion for classification of systemic lupus erythematosus: pitfalls and opportunities. Ann Rheum Dis 2018 Jun 23; doi: 10.1136/annrheumdis-2018-213821.

22. Meroni PL, Bizzaro N, Cavazzana I, Borghi MO, Tincani A. Automated tests of ANA immunofluorescence as throughput autoantibody detection technology: strengths and limitations. BMC 2014;12:38.

23. Tozzoli R, Villalta D, Bizzaro N. Challenges in the standardization of autoantibody testing: a comprehensive review. Clin Rev Allergy Immunol 2017;53:68-77.

24. Van Hoovels L, Schouwers S, Van den Bremt S, Bogaert L, Vandeputte N, Vercammen M, et al. Analytical performance of the single well titer function of NOVA View ${ }^{\circledR}$ : good enough to omit ANA IIF titer analysis? [Letter] Clin Chem Lab Med 2018;56:e258-61.

Supplementary Material: The online version of this article offers supplementary material (https://doi.org/10.1515/cclm-2018-0933). 